\title{
Comments on: The effect of prolonged breast-feeding on the development of postmenopausal osteoporosis in population with insufficient calcium intake and vitamin D level
}

\author{
J. M. Moran ${ }^{1}$ • J. D. Pedrera-Zamorano ${ }^{1}$
}

Received: 12 April 2016 / Accepted: 30 June 2016 / Published online: 11 July 2016

(C) International Osteoporosis Foundation and National Osteoporosis Foundation 2016

Dear Editor,

We have read with interest the paper by Yun and colleagues [1], recently published in Osteoporosis International, about the effect of prolonged breast-feeding on the development of postmenopausal osteoporosis in a population with insufficient calcium intake and vitamin D level. Based on the data provided by the authors we would like to make the following comments:

There is a concern about the distribution of the subjects in this manuscript. A total of 1231 women were included in this study: 304 diagnosed with osteoporosis and 927 that formed the non-osteoporotic control group. However, when grouped by breast-feeding history women were divided into never ( $n=104),<24$ months $(n=451)$ and $\geq 24$ months (673), comprising a total of 1228 women. If this discrepancy means there were a number of women $(n=3)$ with lost breast-feeding history they should be excluded from the study as indicated in Fig. 1 of the paper. If breast-feeding history was lost, then the figures in Table 1 of the paper are also erroneous, as it indicates that a total of 1231 women were analyzed.

Additionally, in Table 2, 96 non-osteoporotic women never breast-fed, compared with 7 women who had osteoporosis in the femoral neck (ie, women with osteoporosis either in the femoral neck alone or in the femoral neck + lumbar spine). If

A response to these comments can be found at doi: http://dx.doi. org/10.1007/s00198-016-3698-0.

\section{J. M. Moran}

jmmorang@unex.es

1 Metabolic Bone Diseases Research Group, University of Extremadura, Cáceres, Spain we then look at the number of women who had osteoporosis in the lumbar spine (which should comprise women with osteoporosis either in the lumbar spine alone or the lumbar spine + femoral neck), we can understand changes in the figures (12 women in Table 2), but how can this change affect the number of non-osteoporotic women that never breast-fed? And, intriguingly, how can the global number of non-osteoporotic women that never breast-fed (bottom of the table) differ from the previous figures? If the authors found that osteoporosis was absent in 89 women that never breast-fed, 368 women that breast-fed for $<24$ months, and 468 women that breast-fed for $\geq 24$ months, it is strange that such figures change across groups in Table 2. We are sincerely of the opinion that the data presented in Table 2 do not only reflect women from the nonosteoporotic group. Overall, and based on the data presented in Table 2 from the Yun and colleagues paper, the figures are 303 (vs 304) osteoporotic women and 925 (vs 927) nonosteoporotic women.

Although these errors were not substantial enough to affect the findings and conclusions, we do not feel that such mistakes should be left uncorrected in a reputable journal.

Compliance with ethical standards

Conflicts of interest Jose M. Moran and Juan D. Pedrera-Zamorano declare that they have no conflict of interest.

\section{Reference}

1. Yun BH, Chon S, Choi YS, Cho S, Lee BS, Seo SK (2016) The effect of prolonged breast-feeding on the development of postmenopausal osteoporosis in population with insufficient calcium intake and vitamin D level. Osteoporos Int. doi:10.1007/s00198-016-3585-8 